\title{
Clinical Application of Computer-Aided Diagnosis for Breast Ultrasonography: Factors That Lead to Discordant Results in Radial and Antiradial Planes
}

\author{
Ying Zhu, Weiwei Zhan, Xiaohong Jia, Juan Liu, Jianqiao Zhou \\ Department of Ultrasound, Shanghai Ruijin Hospital Affiliated to Medical School of Shanghai Jiaotong University, Shanghai, People's Republic of China
}

Correspondence: Jianqiao Zhou, Department of Ultrasound, Shanghai Ruijin Hospital Affiliated to Medical School of Shanghai Jiaotong University, 197 Ruijin Er Road, Shanghai, 200025, People's Republic of China, Email zhousu30@I26.com

Objective: To investigate factors that may lead to discordant results in radial and antiradial planes when applying a computer-aided diagnostic system (S-Detect ${ }^{\mathrm{TM}}$ ) for breast ultrasound (US).

Methods: From May 2019 to September 2019, a total of 288 breast lesions from 286 consecutive women were analyzed. Diagnostic performance and diagnostic agreement of the CAD system between the radial and antiradial planes were calculated. Based on the CAD results in the radial and antiradial planes, the lesions were classified into two groups: the agreement group and the discordant group. Ultrasound imaging and clinicopathologic factors in the two groups were compared.

Results: Of the 288 breast lesions, 187 (64.7\%) were benign, and 101 (35.3\%) were malignant. There was no difference in diagnostic performance of the CAD system between the radial and antiradial planes. The diagnostic agreement of the CAD system between these two orthogonal planes was good $(\mathrm{K}=0.645)$. Compared to category $3,4 \mathrm{C}$ and 5 lesions, category $4 \mathrm{~A}$ and $4 \mathrm{~B}$ lesions were more likely to have discordant $\mathrm{CAD}$ results. In the subgroup of malignant tumors, lesions diagnosed as carcinoma in situ $(\mathrm{P}=0.014)$, staged as $\mathrm{T} 1$ $(\mathrm{P}=0.013)$ and with low Ki-67 status $(\mathrm{P}=0.024)$ were significantly associated with discordant CAD results.

Conclusion: The CAD system for breast ultrasound has a favorable diagnostic performance, and discordant CAD results between the radial and antiradial planes were more frequent for BI-RADS category 4A-4B lesions and less invasive malignant lesions.

Keywords: ultrasound, breast, computer-aided diagnosis, agreement

\section{Introduction}

Recently, the occurrence of breast cancer has been increasing in both developed and developing countries. ${ }^{1}$ Early diagnosis can improve the prognosis of breast cancer patients, and breast ultrasound (US) plays an important role in detecting breast cancer. As a convenient, cost-effective, and widely available diagnostic tool, conventional US is able to achieve a sensitivity greater than $90 \% .{ }^{2,3}$ Nevertheless, this method is limited by its high dependence on operator experience. $^{4,5}$

In recent years, artificial intelligence (AI) technology has been greatly developed. Preliminary studies have shown a wide AI application in imaging the lung, thyroid, fetus, carotid, liver, etc. Several deep learning technologies for ultrasound computer-aided diagnosis systems (CADs) have been frequently utilized in investigations to provide a second objective opinion to assist radiologists in image interpretation and diagnosis. ${ }^{6}$ Among them, a deep learning-based CAD system for breast US, also known as S-Detect (Samsung Medison Co. Ltd., Seoul, Korea), has become commercially available. ${ }^{7}$ This system can analyze the morphologic features of a target breast mass automatically and quickly provide a final diagnosis in a dichotomic form of "possibly benign" or "possibly malignant". This system has high diagnostic performance in the diagnosis of breast lesions and is distinguished for its high specificity. ${ }^{8-13}$ Many previous studies have demonstrated that adding S-Detect to breast US can improve the specificity, positive predictive value, and accuracy in the diagnosis of breast lesions and can reduce variability in radiologist interpretations..$^{8-10,13}$ 
Breast tumors often show different morphologies on grayscale images in different examination planes due to their intratumoral heterogeneity and three-dimensional structures, which leads to a high probability that the CAD system would display different results in different acquisition planes when being applied. ${ }^{14,15}$ Currently, to the best of our knowledge, there are no standard guidelines for CAD systems with regard to image acquisition planes. In previous studies, ${ }^{8,13}$ a single representative image of the breast tumor was selected for CAD evaluation. However, the high interobserver variability in the acquisition of representative images seemed inevitable. In some other studies, ${ }^{9,11}$ there was no description of whether the CAD examination was performed in the antiradial plane or the radial plane when classifying the tumors as "possibly malignant". Thus, to decrease the difficulties in interpreting CAD results in clinical practice, it is necessary to assess whether and in what circumstances the orientation of imaging acquisition influences the diagnostic performances of CAD systems.

According to the American College of Radiology practice guidelines for breast US, image acquisition in two orthogonal planes is recommended as a standard procedure to achieve better lesion features. ${ }^{16}$ Therefore, we used a CAD system (S-Detect) to evaluate the frequency of discordant CAD results in the radial and antiradial planes when differentiating breast lesions and to investigate the imaging and clinicopathologic factors that lead to the discordant results.

\section{Patients and Methods}

\section{Patients}

This prospective study, carried out from May 2019 to September 2019, was approved by the Ethics Committee of the Shanghai Ruijin Hospital of Huangpu District. Written informed consent was obtained from the patients or parents of patients under 18 years of age, and this study was conducted in compliance with the Declaration of Helsinki. Three hundred thirty breast lesions in 328 consecutive patients who were scheduled for ultrasound-guided core needle biopsy or ultrasound-guided vacuum-assisted excision were included in this study. Among them, 42 patients were excluded from the study for the following reasons: 7 patients had nonmass lesions on ultrasound imaging; 19 lesions were currently being treated with neoadjuvant chemotherapy; 9 lesions were larger than $4 \mathrm{~cm}$; 4 lesions were biopsied before the US examination; and 3 women were pregnant or lactating. Finally, the final cohort for this study included 288 breast lesions in 286 women (mean age, $47.3 \pm 13.8$ years; age range, 19-86 years) (Figure 1). One hundred and twenty-six patients (44.1\%) were asymptomatic, while the remaining 160 patients $(55.9 \%)$ presented with symptoms such as a palpable mass, breast pain and bloody nipple discharge.

\section{Conventional Ultrasound Examinations and the Application of the Computer-Aided Diagnosis System}

An RS80A system (Samsung Medison Co., Ltd.) equipped with a deep learning-based CAD system software for breast (S-Detect; Samsung Medison, Co. Ltd., Seoul, Korea) was applied for breast imaging with a 5-12 MHz linear highfrequency transducer. To remove any bias arising from multiple operators performing CAD and reduce interobserver variability, the examinations of the study population were performed by a single expert radiologist (J.Q.Z) with 18 years of experience in breast US imaging who has performed more than 100 CAD examinations.

Informed of the patients' clinical information and mammography results, the radiologist performed the bilateral breast US examinations using the routine scanning protocol. When the target lesion was detected, Figure 2 radial and antiradial static images (Figure 2), radial and antiradial video clips, representative color Doppler images were recorded for further imaging evaluation. After the conventional US examination, the S-Detect examination was performed for the static images in the radial and antiradial planes by the same radiologist who performed the breast US examination. When the image was selected, a region of interest (ROI) was automatically drawn along the lesion margin by the S-detect software. If the automatically generated ROI was considered inaccurate by the radiologist, it was manually adjusted. After the ROI was delineated, the final evaluations from the CAD system were displayed in dichotomized form as "probably benign" or "possibly malignant". 


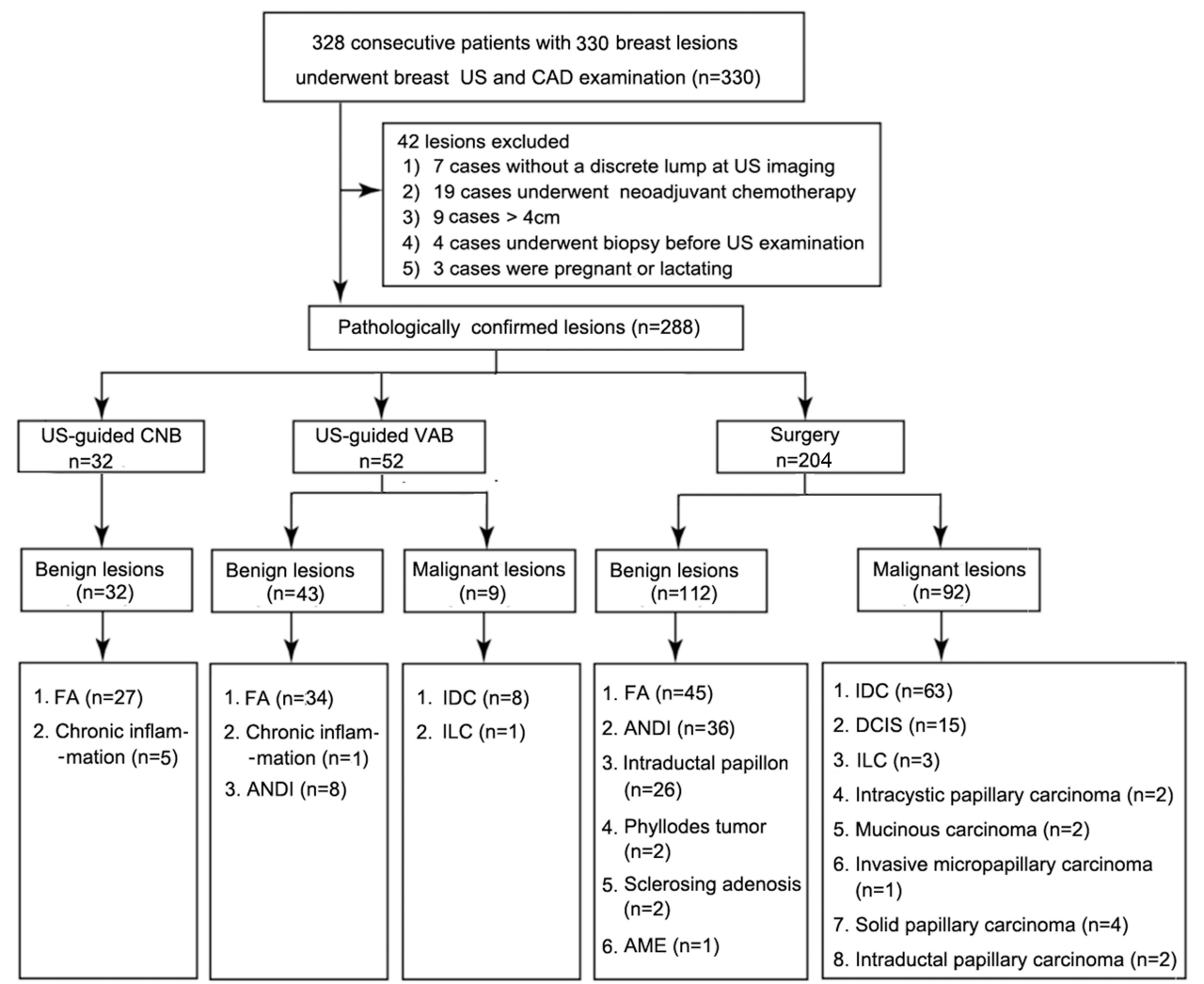

Figure I Flowchart of the breast lesion selection process.

Abbreviations: CAD, computer-aided diagnosis; CNB, core needle biopsy; VAB, vacuum-assisted biopsy; FA, fibroadenoma; ANDI, aberrations of normal development and involution without fibroadenoma; IDC, invasive ductal carcinoma; ILC, invasive lobular carcinoma; AME, adenomyoepithelioma; DCIS, ductal carcinoma in situ.

A

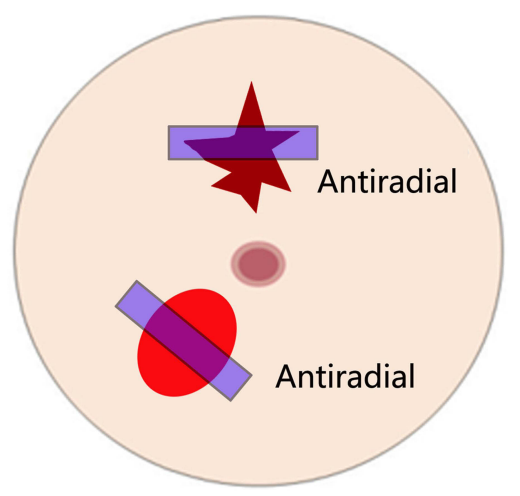

B

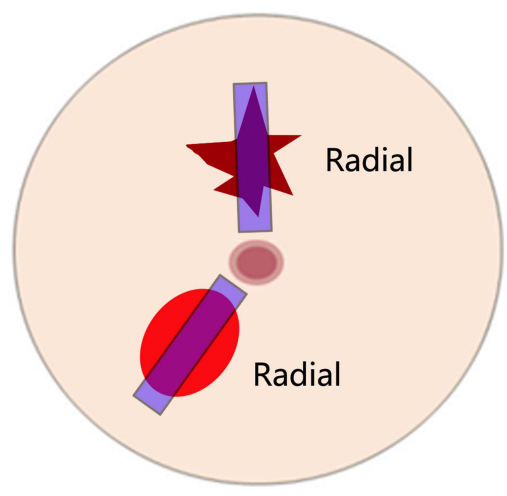

Figure 2 Image acquisition directions for the breast lesions. (A) Antiradial plane; (B) radial plane.

\section{Imaging Review}

Two radiologists (Y.Z. and J.X.H) with more than 8 years of experience in breast US were asked to review the static images and video clips of the breast lesions. They had no access to the patients' clinical information or CAD results but were aware of the patients' age. If disagreements occurred, they jointly reviewed the images, discussed the findings, and reached a consensus. The US features, including shape, orientation, margin, echo pattern, posterior features, calcifications, changes in the surrounding tissue, degree of blood flow, tumor location and tissue composition of the breast gland adjacent to the lesions, were described. In this study, the degree of blood flow in the breast lesions was divided as follows according to the grading by Adler et $\mathrm{al}^{17}$ absent, no blood flow was visualized; minimal, 1 or 2 pixels containing blood 
flow were visualized; moderate, a main vessel and several small vessels were visualized; and marked, 4 or more vessels were visualized. Finally, each target lesion was classified as category 3, 4A, 4B, 4C, or 5 according to the fifth edition of the Breast Imaging Reporting and Data System (BI-RADS) lexicon.

\section{Statistical Analysis}

Statistical analyses were performed by using SPSS version 16.0 (SPSS Inc, Chicago, IL) and MedCalc for Windows, version 12.2.0.0 (MedCalc Software, Mariakerke, Belgium). Descriptive variables are presented as the mean \pm SD. Categorical variables are described as proportions. Using histologic results as the reference, the diagnostic sensitivity, specificity, and area under the ROC curve (AUC) of the CAD system were calculated. The kappa coefficient (k) was calculated to determine the agreement between CAD results in the radial and antiradial planes. The $\mathrm{k}$ values were interpreted as follows using the guidelines of Landis and Koch: ${ }^{18}$ poor agreement, $\mathrm{k}=0.00-0.20$; fair agreement, $\mathrm{k}=0.21-0.40$; moderate agreement, $\mathrm{k}=0.41-0.60$; good agreement $\mathrm{k}=0.61-0.80$; and excellent agreement, $\mathrm{k}=0.81-$ 1.00. Based on the CAD results in the radial and antiradial planes, the lesions were classified into two groups: the agreement group, in which the tumor had the same CAD results in the two orthogonal planes; and the discordant group, in which the tumor had different CAD results in the two orthogonal planes. Chi-square tests and Fisher's exact tests were applied to compare the categorical variables. Statistical significance was determined at a $\mathrm{P}$ value $<0.05$.

\section{Results}

\section{Demographic Data and Histologic Findings}

Of the 288 breast lesions, 187 (64.7\%) were benign, and 101 (35.3\%) were malignant. The malignant lesions were significantly larger than the benign lesions: $18.42 \pm 7.48 \mathrm{~mm}(9.00-40.00 \mathrm{~mm})$ vs $15.68 \pm 7.60 \mathrm{~mm}(7.00-39.10 \mathrm{~mm})$ $(\mathrm{P}=0.004)$. The patients with malignant lesions were significantly older than those with benign lesions (mean age: $55.9 \pm$ 12.9 years vs $42.4 \pm 11.7$ years, $\mathrm{P}<0.001)$. The pathological results of the breast lesions are shown in Figure 1.

\section{Diagnostic Agreement of the CAD Evaluations in the Radial and Antiradial Planes}

Among the 288 lesions, 105 cases had the same CAD results of "possibly malignant" and 131 cases had the same CAD results of "probably benign" lesions in the radial and antiradial planes, while 52 cases had discordant CAD results, including 22 cases with "possibly malignant" evaluations in only the antiradial plane and 30 cases with "possibly malignant" evaluations in only the radial plane. The diagnostic agreement of the CAD results based on the images in the radial and antiradial planes was good, with a $\mathrm{K}$ value of 0.645 (95\% CI: 0.557-0.733). In addition, compared with those based on the images in the antiradial planes, CAD evaluations based on the ultrasound images in the radial planes had a similar sensitivity $(79.4 \%$ vs $85.3 \%)$, specificity $(75.4 \%$ vs $72.2 \%)$ and AUC $(0.774$ vs 0.790$)$ in predicting malignant lesions ( $\mathrm{P}>0.05$ for all).

\section{Correlation with Ultrasound Features}

There was a significant association between BI-RADS category and discordant CAD results for breast lesions in the radial and antiradial planes $(\mathrm{P}=0.011)$. Our data showed that the rates of malignancy were $3.20 \%$ (three of 103 ) for category 3 lesions, 12.2\% (10 of 82) for category 4A lesions, 59.6\% (28 of 47) for category 4B lesions, 88.9\% (five of 45 ) for category $4 \mathrm{c}$ lesions, and $100 \%$ (21 of 21 ) for category 5 lesions. Compared to category 3, 4C and 5 lesions, category $4 \mathrm{~A}$ and $4 \mathrm{~B}$ lesions were more likely to have discordant $\mathrm{CAD}$ results in the two orthogonal planes (Table 1). In this study, 127 lesions were categorized as BI-RADS 4A or 4B, and $34(26.8 \%)$ of these had discordant CAD results (Figures 3 and 4).

During the application of S-Detect, a region of interest (ROI) along the border of the mass was drawn automatically by the CAD system or was adjusted manually by the radiologist. We did not find that the radiologist's adjustments to the ROI led to bias in the final CAD results. In this study, the radiologist manually adjusted the ROIs in 69 (24.0\%) cases, including 25 benign tumors and 44 malignant tumors. Among them, only one case proven to be DCIS had a changed CAD result of "possibly malignant" in the radial plane. The other cases had no changes in CAD results before and after 
Table I Relationship of Agreement of CAD Results in Orthogonal Planes with Pathology Findings and Imaging Features

\begin{tabular}{|c|c|c|c|}
\hline & $\begin{array}{l}\text { Agreement Group } \\
(n=236)\end{array}$ & $\begin{array}{l}\text { Discordant Group } \\
(n=52)\end{array}$ & p value \\
\hline Age & & & 0.429 \\
\hline$<50 y(n=180)$ & I50 (83.3\%) & $30(16.7 \%)$ & \\
\hline$\geq 50 y(n=108)$ & $86(79.6 \%)$ & $22(20.4 \%)$ & \\
\hline Pathology & & & 0.126 \\
\hline Benign $(n=\mid 87)$ & I 58 (84.5\%) & 29 (I5.5\%) & \\
\hline Malignant $(n=101)$ & 78 (77.2\%) & $23(15.5 \%)$ & \\
\hline Manually adjusted ROI & & & 0.580 \\
\hline Yes $(n=69)$ & 55 (79.7\%) & $14(20.3 \%)$ & \\
\hline No $(n=219)$ & I8I (82.6\%) & 38 (17.4\%) & \\
\hline Size & & & 0.664 \\
\hline$\leq 20 \mathrm{~mm}(\mathrm{n}=209)$ & $170(8 \mid .3)$ & 39 (I8.7\%) & \\
\hline$>20 \mathrm{~mm}(\mathrm{n}=79)$ & 66 (83.5\%) & $13(16.5 \%)$ & \\
\hline Location & & & 0.315 \\
\hline Upper outer quadrant & $104(83.2 \%)$ & $21(16.8 \%)$ & \\
\hline Lower outer quadrant & $41(87.2 \%)$ & $6(12.8 \%)$ & \\
\hline Lower inner quadrant & 29 (78.4\%) & $8(21.6 \%)$ & \\
\hline Upper inner quadrant & 48 (75.0\%) & $16(25.0 \%)$ & \\
\hline Central quadrant & 14 (93.3\%) & I (6.7\%) & \\
\hline Tissue composition & & & 0.109 \\
\hline Homogeneous background echotexture - fat $(n=37)$ & $26(70.3 \%)$ & II (29.7\%) & \\
\hline Homogeneous background echotexture - fibroglandular $(n=91)$ & $74(81.3 \%)$ & 17 (18.7\%) & \\
\hline Heterogeneous background echotexture $(n=160)$ & $136(81.9 \%)$ & $24(15.0 \%)$ & \\
\hline Shape & & & 0.324 \\
\hline Oval $(n=119)$ & $102(85.7 \%)$ & $17(14.3 \%)$ & \\
\hline Round $(n=7)$ & $5(71.4 \%)$ & $2(28.6 \%)$ & \\
\hline Irregular $(n=162)$ & $129(79.6 \%)$ & $33(20.4 \%)$ & \\
\hline Orientation & & & 0.075 \\
\hline Parallel (n=257) & 207 (80.5\%) & $50(19.5 \%)$ & \\
\hline Not parallel $(n=31)$ & $29(93.5 \%)$ & $2(6.5 \%)$ & \\
\hline Margin & & & 0.110 \\
\hline Circumscribed $(n=117)$ & 101 (86.3\%) & $16(13.7 \%)$ & \\
\hline Not circumscribed $(n=|7|)$ & 135 (78.9\%) & $36(21.1 \%)$ & \\
\hline Echo pattern & & & 0.717 \\
\hline Hypoechoic $(n=256)$ & $211(82.4 \%)$ & $45(17.6 \%)$ & \\
\hline Hyperechoic $(n=16)$ & $12(75.0 \%)$ & $4(25.0 \%)$ & \\
\hline Complex cystic and solid $(n=14)$ & $12(85.7 \%)$ & $2(14.3 \%)$ & \\
\hline Heterogeneous $(n=2)$ & I (50\%) & I (50\%) & \\
\hline Posterior features & & & 0.546 \\
\hline None $(n=\mid 82)$ & I 47 (80.8\%) & 35 (19.2\%) & \\
\hline Shadowing $(n=27)$ & $23(85.2 \%)$ & $4(14.8 \%)$ & \\
\hline Enhancement $(n=7 I)$ & $58(81.7 \%)$ & $13(18.3 \%)$ & \\
\hline Combined pattern $(n=8)$ & $8(100 \%)$ & $0(0 \%)$ & \\
\hline Microcalcifications in the mass & & & 0.177 \\
\hline Present $(n=52)$ & $46(88.5 \%)$ & $6(11.5 \%)$ & \\
\hline Absent $(n=236)$ & 190 (80.5\%) & $46(19.5 \%)$ & \\
\hline Degree of vascularity in the mass & & & 0.193 \\
\hline Absent $(n=103)$ & $85(81.6 \%)$ & $19(18.4 \%)$ & \\
\hline Minimal $(n=55)$ & $40(72.7 \%)$ & $15(27.3 \%)$ & \\
\hline Moderate $(n=7 I)$ & $61(85.9 \%)$ & $10(14.1 \%)$ & \\
\hline Marked $(n=59)$ & $5 \mathrm{I}(86.4 \%)$ & $8(13.6 \%)$ & \\
\hline
\end{tabular}

(Continued) 
Table I (Continued).

\begin{tabular}{|l|l|l|l|}
\hline & $\begin{array}{l}\text { Agreement Group } \\
(\mathbf{n = 2 3 6 )}\end{array}$ & $\begin{array}{l}\text { Discordant Group } \\
(\mathbf{n = 5 2})\end{array}$ & P value \\
\hline $\begin{array}{l}\text { Changes in the surrounding tissue } \\
\text { Present }(n=53)\end{array}$ & $47(88.7 \%)$ & $6(11.3 \%)$ & 0.158 \\
Absent (n=235) & $189(80.4 \%)$ & $46(19.6 \%)$ & \\
US-based BI-RADS category & $85(86.7 \%)$ & $13(13.3 \%)$ & 0.011 \\
$3(n=98)$ & $63(75.0 \%)$ & $21(25.0 \%)$ & \\
$4 A(n=84)$ & $30(69.8 \%)$ & $13(30.2 \%)$ & \\
$4 B(n=43)$ & $38(90.5 \%)$ & $4(9.5 \%)$ & \\
$4 C(n=42)$ & $20(95.2 \%)$ & $1(4.8 \%)$ & \\
$5(n=21)$ & & \\
\hline
\end{tabular}

Note: Values are presented as number (\%).

adjusting the ROI for CAD analysis. Accordingly, the adjustment of the ROIs was not related to the agreement of the CAD results between the two orthogonal planes (Table 1). No correlation was found among the discordant CAD results, tumor size on ultrasonography, tissue composition of the breast gland adjacent to the lesions and other ultrasound features of the breast lesions (Table 1).

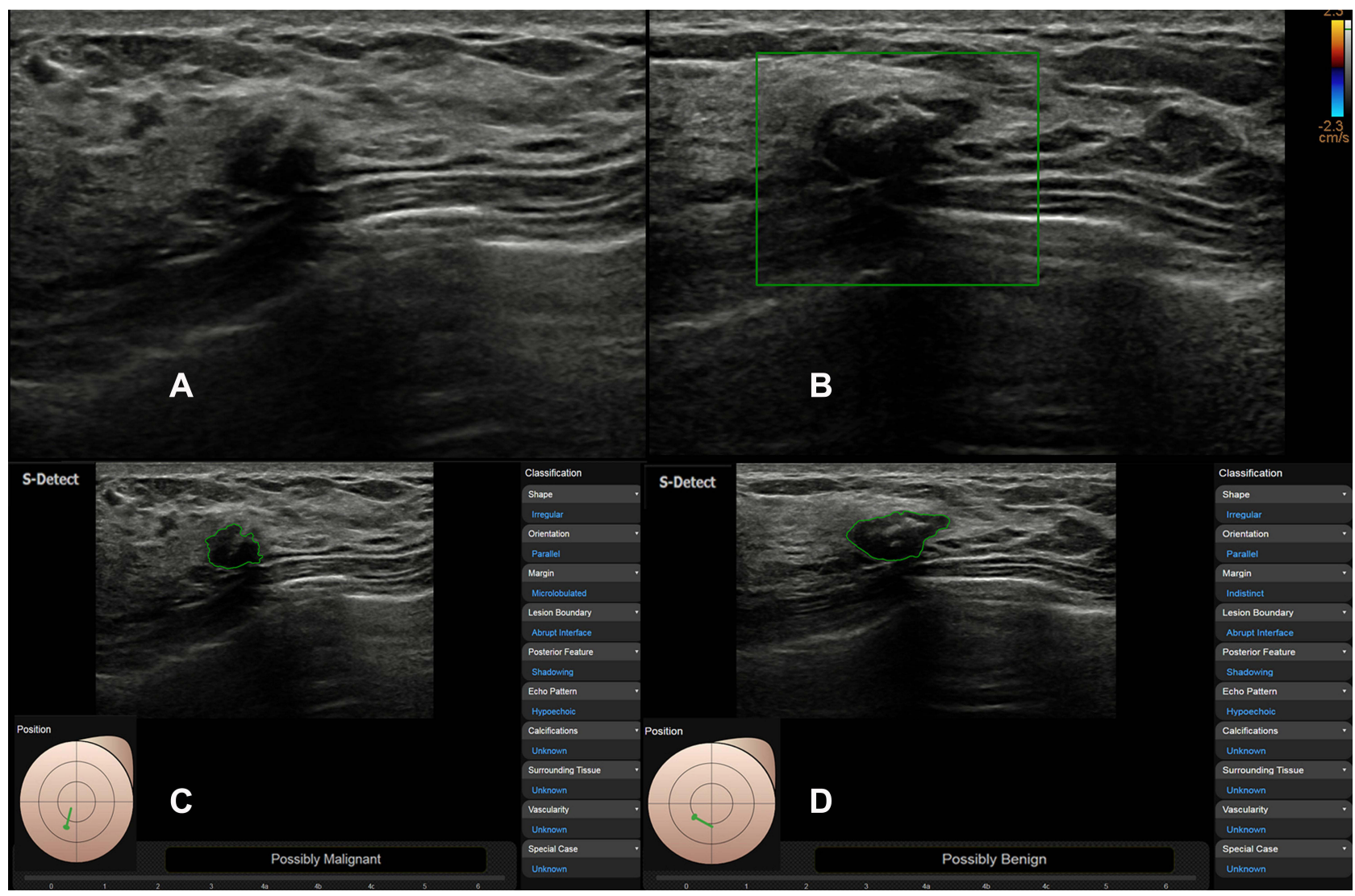

Figure 3 US images in a 40-year-old woman with fibroadenoma. Conventional ultrasound images show 13-mm hypoechoic mass with irregular shape and macrocalcification $(\mathbf{A}$ and B), which was classified as BI-RADS 4A. The CAD system diagnosed it as "possibly malignant" based on the image in the radial plane (C) and "probably benign" based on the image in the antiradial plane (D). 


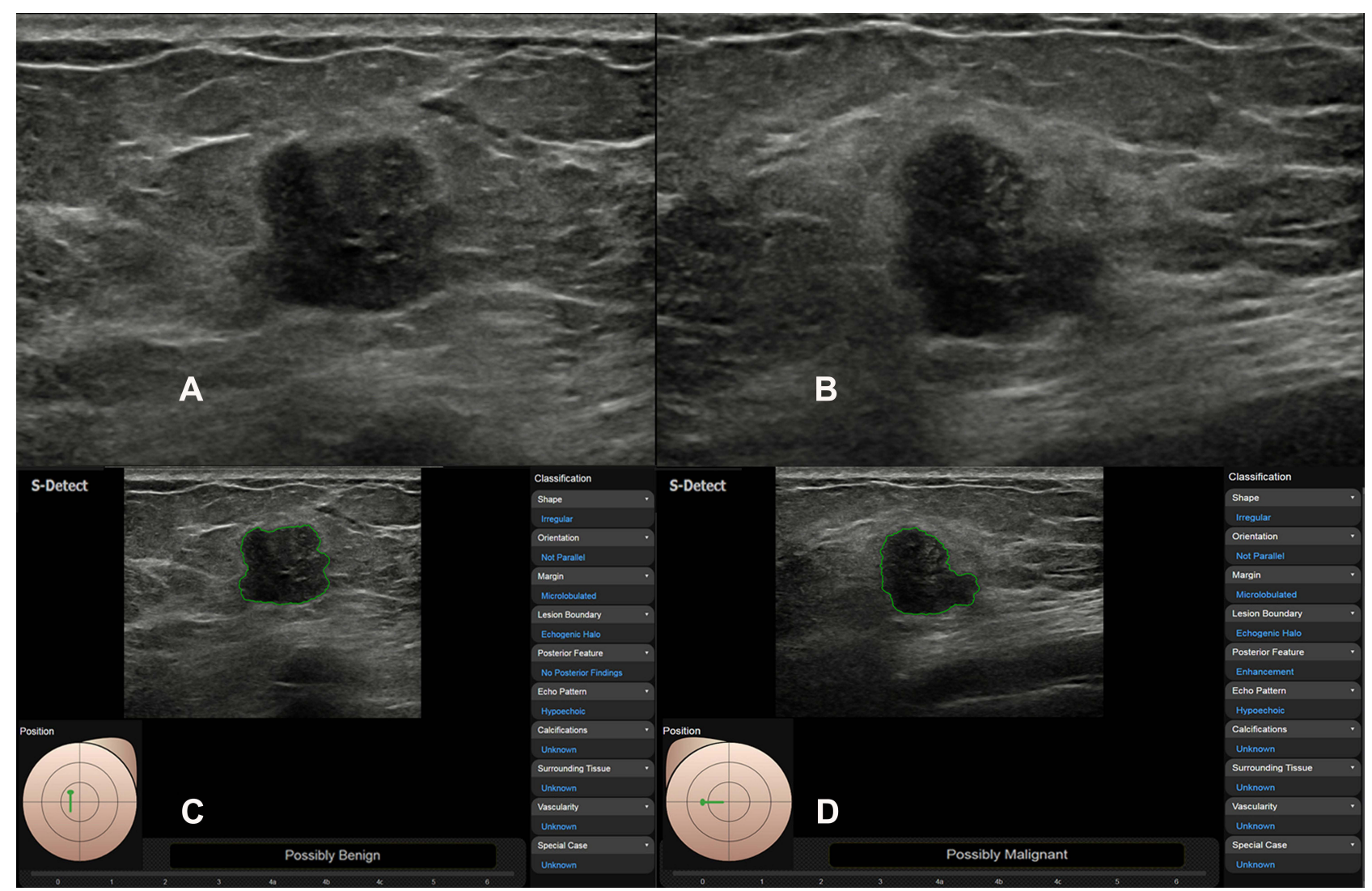

Figure 4 US images in a 54-year-old woman with Her-2 positive invasive ductal carcinoma. Conventional ultrasound images show I5-mm hypoechoic mass with irregular shape (A and B), which was classified as BI-RADS 4B. The CAD system diagnosed it as "probably benign" based on the image in the antiradial plane (C) and "possibly malignant" based on the image in the radial plane (D).

\section{Correlation with Clinicopathologic Features}

In this study, the risk of malignancy and patient age showed no significant differences between the agreement and discordant group. However, in the subgroup of malignant tumors, discordant $\mathrm{CAD}$ results between the two orthogonal planes were more frequent among lesions proven as carcinoma in situ by pathology $(\mathrm{P}=0.014)$, staged as $\mathrm{T} 1(\mathrm{P}=0.013)$ and with low Ki-67 status ( $\mathrm{P}=0.024)$ (Table 2), and the discordant results were not related to the expression of $\mathrm{ER}$, $\mathrm{PR}$ or CerBb-2.

\section{Discussion}

At present, CAD systems for breast ultrasound are considered to have good prospects for wide clinical application. ${ }^{19-21}$ S-Detect (Samsung Medison Co. Ltd., Seoul, Korea) is a deep learning-based CAD system that is commercially available and has become one of the most increasingly used CAD systems for the diagnosis of breast cancer. Consistent with previous studies, ${ }^{8-12}$ our study confirmed that this CAD system exhibited a favorable diagnostic value in assisting physicians to discriminate benign and malignant breast lesions, with a sensitivity of $79.4 \%-85.3 \%$, a specificity of $72.7 \%$ $75.4 \%$ and an AUC of $0.774-0.790$.

Because of their heterogeneous and three-dimensional structures, breast tumors might have different CAD results in different scanning planes. ${ }^{14,15}$ After a thorough evaluation of the literature, it appears that our study is the first to evaluate the agreement of CAD results between two orientations of image acquisition. Our data showed good agreement $(\mathrm{k}=0.645)$ between the $\mathrm{CAD}$ results for lesions in the antiradial and radial planes. We also found that the diagnostic performance of S-Detect based on ultrasound images in the antiradial plane did not significantly differ from that based on images in the radial plane. Even so, a better understanding of the factors related to discordant CAD findings between the 
Table 2 Relationship Between Agreement of CAD Results in the Orthogonal Planes and Pathology Findings Among Malignant Lesions $(n=101)$

\begin{tabular}{|c|c|c|c|}
\hline & Agreement Group ( $n=78)$ & Disagreement Group (n=23) & p value \\
\hline Size & & & 0.013 \\
\hline$\leq 20 \mathrm{~mm}(\mathrm{n}=66)$ & $46(69.7 \%)$ & $20(30.3 \%)$ & \\
\hline$>20 \mathrm{~mm}(\mathrm{n}=35)$ & 32 (91.4\%) & $3(8.6 \%)$ & \\
\hline Pathological type & & & 0.014 \\
\hline Carcinoma in situ $(n=2 I)$ & $12(57.1 \%)$ & 9 (42.9\%) & \\
\hline Invasive cancer $(\mathrm{n}=80)$ & $66(82.5 \%)$ & 14 (I7.5\%) & \\
\hline ER and/or PR & & & 0.168 \\
\hline Negative $(n=34)$ & $29(85.3 \%)$ & $5(14.7 \%)$ & \\
\hline Positive $(n=67)$ & 49 (73.1\%) & 18 (26.9\%) & \\
\hline CerbB-2 & & & 0.510 \\
\hline 0 or $1+(n=38)$ & $28(73.7 \%)$ & $10(26.3 \%)$ & \\
\hline $2++$ or $3+++(n=63)$ & $50(79.4 \%)$ & $13(20.6 \%)$ & \\
\hline Ki67 status & & & 0.024 \\
\hline Low $(<14 \% ; n=37)$ & $24(64.9 \%)$ & $13(35.1 \%)$ & \\
\hline High $(\geq 14 \% ; n=64)$ & $54(84.4 \%)$ & $10(15.6 \%)$ & \\
\hline
\end{tabular}

Note: Values are presented as number (\%).

two orthogonal planes might be helpful in the use of this CAD system and the interpretation of its results. Therefore, in this study, we analyzed the clinicopathologic and imaging features of the lesions related to discordant CAD results.

Although malignant breast lesions appear more likely to have complicated and variable intratumor heterogeneity than benign lesions, ${ }^{14}$ we did not find a significant correlation between discordant CAD results between the two orthogonal planes and tumor pathology. Notably, our data showed that malignant lesions diagnosed as carcinoma in situ, with a smaller size and with low Ki-67 status were more likely to have discordant CAD results in the orthogonal planes. The main reason might be related to their different growth rates in different directions and their high frequency for displaying nonspecific US features. ${ }^{22,23}$ This subgroup analysis indicated that the observed disagreement for CAD results of malignant lesions may correlate with the invasive potential of the lesion. Of course, this has to be proven further in a large-scale study.

Moreover, our results found that lesions categorized as BI-RADS 4A or 4B were more likely to have different CAD results between two orthogonal planes. Previous studies have comfirmed that radiologists had an improved specificity, positive predictive value (PPV) and accuracy after integrating CAD results into their initial evaluations. ${ }^{9-12}$ But according to our experience, they might encounter difficulties in integrating CAD findings into their initial conventional ultrasound results when considering images of breast lesions from two orthogonal view rather than form a single view. Considering that there are no specific guidelines on the application of CAD for the breast, this decision might inevitably lead to high heterogeneity when discordant CAD results are obtained for BI-RADS category 4A and 4B lesions. Thus, the optimal strategy for adding CAD results to improve radiologists' diagnostic performance needs to be clarified.

The CAD system of S-Detect can can perform autosegmentation and interpretation based on US-based morphological descriptions. However, before the classification of lesions is displayed by the S-Detect system, radiologists need to delineate the ROI. The ROI for CAD analysis is not automatically segmented in the full sense and needs to be adjusted manually if the outline is not adequate. Jeong et $\mathrm{al}^{24}$ found that radiologists with different experience levels had different accuracies in applying the S-Detect system for the thyroid because of differences in the identified ROI. In contrast, we found that manual adjustment of the ROI by an experienced radiologist had little influence on the results of this CAD system and accordingly had little effect on the agreement of the CAD results between two orthogonal planes. In this study, the ROI was manually adjusted in $24.0 \%$ of cases (69/288). Among them, only one case proven to be DCIS had a changed CAD result in the radial plane as "possibly malignant". The main reasons might be as follows: 1) the differences in the outline detected by the CAD system and the manual adjustments were typically small; ${ }^{25}$ and 2 ) this 
CAD system focuses not only on the ROI area but also on the peripheral area within 180 pixels when analyzing the ultrasound image of breast lesions, ${ }^{7}$ which might reduce the influence of manual adjustments on the core analysis area. Moreover, other potential factors that preclude the application of this CAD system for evaluating breast lesions, including changes in the surrounding tissue, microcalcifications and degree of vascularity in the mass, were also discussed in this study, and we did not find any correlations with discordant CAD results between two orthogonal planes.

There were a few limitations in our study. First, this study was a single-center, retrospective study, although care was taken to minimize bias by blinding the radiologists to the final pathology of the lesions, and the radiologists performing the BI-RADS classification were did not perform the CAD examination. Second, there was a risk for potential bias with respect to data collection, as only biopsy-confirmed lesions were included. Therefore, lesions categorized as BI-RADS 2 were not included. Third, this study was solely based on an investigation with one CAD system (S-Detect), and the results might vary with other systems. In the future, the performance of deep learning-based CAD systems may be improved with technical developments, especially when CAD systems based on the video clips rather than static images are developed. However, even then, an evaluation of discordant CAD findings between radial and antiradial planes would still be of interest.

In conclusion, the deep learning-based CAD system (S-Detect) is an effective tool for aiding US-based diagnosis of breast lesions and have a favorable diagnostic performance. The agreement between the CAD results based on the radial and antiradial planes was good. Discordant CAD results were more frequent for lesions categorized as BI-RADS $4 \mathrm{a}$ or $4 \mathrm{~b}$ and for less invasive malignant lesions. The recognition of discordant CAD results can help to avoid inaccurate evaluations of breast cancer with low invasiveness and decrease the difficulty in interpreting CAD results in clinical practice.

\section{Disclosure}

The authors report no conflicts of interest in this work.

\section{References}

1. Ferlay J, Soerjomataram I, Dikshit R, et al. Cancer incidence and mortality worldwide: sources, methods and major patterns in GLOBOCAN 2012. Int J Cancer. 2015;136(5):E359-E386. doi:10.1002/ijc.29210

2. Kolb TM, Lichy J, Newhouse JH. Comparison of the performance of screening mammography, physical examination, and breast US and evaluation of factors that influence them: an analysis of 27,825 patient evaluations. Radiology. 2002;225(1):165-175. doi:10.1148/radiol.2251011667

3. Berg WA, Zhang Z, Lehrer D, et al. Detection of breast cancer with addition of annual screening ultrasound or a single screening MRI to mammography in women with elevated breast cancer risk. JAMA. 2012;307(13):1394-1404. doi:10.1001/jama.2012.388

4. Abdullah N, Mesurolle B, El-Khoury M, Kao E. Breast imaging reporting and data system lexicon for US: interobserver agreement for assessment of breast masses. Radiology. 2009;252(3):665-672. doi:10.1148/radiol.2523080670

5. Lazarus E, Mainiero MB, Schepps B, Koelliker SL, Livingston LS. BI-RADS lexicon for US and mammography: interobserver variability and positive predictive value. Radiology. 2006;239(2):385-391. doi:10.1148/radiol.2392042127

6. Doi K. Computer-aided diagnosis in Medical imaging: historical review, current status and future potential. Comput Med Imaging Graph. 2007;31 (4-5):198-211. doi:10.1016/j.compmedimag.2007.02.002

7. Han S, Kang HK, Jeong JY, et al. A deep learning framework for supporting the classification of breast lesions in ultrasound images. Phys Med Biol. 2017;62(19):7714-7728. doi:10.1088/1361-6560/aa82ec

8. Choi JH, Kang BJ, Baek JE, Lee HS, Kim SH. Application of computer-aided diagnosis in breast ultrasound interpretation: improvements in diagnostic performance according to reader experience. Ultrasonography. 2018;37(3):217-225. doi:10.14366/usg.17046

9. Cho E, Kim EK, Song MK, Yoon JH. Application of computer-aided diagnosis on breast ultrasonography: evaluation of diagnostic performances and agreement of radiologists according to different levels of experience. J Ultrasound Med. 2018;37(1):209-216. doi:10.1002/jum.14332

10. Di Segni M, de Soccio V, Cantisani V, et al. Automated classification of focal breast lesions according to S-detect: validation and role as a clinical and teaching tool. $J$ Ultrasound. 2018;21(2):105-118. doi:10.1007/s40477-018-0297-2

11. Zhao C, Xiao M, Jiang Y, et al. Feasibility of computer-assisted diagnosis for breast ultrasound: the results of the diagnostic performance of S-detect from a single center in China. Cancer Manag Res. 2019;11:921-930. doi:10.2147/CMAR.S190966

12. Kim K, Song MK, Kim EK, Yoon JH. Clinical application of S-Detect to breast masses on ultrasonography: a study evaluating the diagnostic performance and agreement with a dedicated breast radiologist. Ultrasonography. 2017;36(1):3-9. doi:10.14366/usg.16012

13. Choi JS, Han BK, Ko ES, et al. Effect of a deep learning framework-based computer-aided diagnosis system on the diagnostic performance of radiologists in differentiating between malignant and Benign masses on breast ultrasonography. Korean J Radiol. 2019;20(5):749-758. doi: $10.3348 / \mathrm{kjr} .2018 .0530$

14. Marusyk A, Almendro V, Polyak K. Intra-tumour heterogeneity: a looking glass for cancer. Nat Rev Cancer. 2012;12(5):323-334. doi:10.1038/ $\operatorname{nrc} 3261$

15. Polyak K, Vogt PK. Progress in breast cancer research. Proc Natl Acad Sci U S A. 2012;109(8):2715-2717. doi:10.1073/pnas.1201091109 
16. Whitman G, Rapp C, Piccoli Cet al. AIUM practice guideline for the performance of a breast ultrasound examination. J Ultrasound Med. 2009;28 (1):105-109. doi:10.7863/jum.2009.28.1.105

17. Adler DD, Carson PL, Rubin JM, Quinn-Reid D. Doppler ultrasound color flow imaging in the study of breast cancer: preliminary findings. Ultrasound Med Biol. 1990;16(6):553-559. doi:10.1016/0301-5629(90)90020-D

18. McMilin C, Camargo C, Morton S, Grant C. Understanding administrative coding of emergency Department visits for unspecified acute allergic reactions. N Z Med J. 2015;128(1421):39-46.

19. Hu Q, Giger ML. Clinical artificial intelligence applications: breast imaging. Radiol Clin North Am. 2021;59(6):1027-1043. doi:10.1016/j. rcl.2021.07.010

20. Shan J, Alam SK, Garra B, Zhang Y, Ahmed T. Computer-aided diagnosis for breast ultrasound using computerized BI-RADS features and machine learning methods. Ultrasound Med Biol. 2016;42(4):980-988. doi:10.1016/j.ultrasmedbio.2015.11.016

21. Tan T, Platel B, Twellmann T, et al. Evaluation of the effect of computer-aided classification of benign and malignant lesions on reader performance in automated three-dimensional breast ultrasound. Acad Radiol. 2013;20(11):1381-1388. doi:10.1016/j.acra.2013.07.013

22. Thomson JZ, Evans AJ, Pinder SE, Burrell HC, Wilson AR, Ellis IO. Growth pattern of ductal carcinoma in situ (DCIS): a retrospective analysis based on mammographic findings. Br J Cancer. 2001;85(2):225-227.

23. Wu JY, Zhao ZZ, Zhang WY, et al. Computer-aided diagnosis of solid breast lesions with ultrasound: factors associated with false-negative and false-positive results. $J$ Ultrasound Med. 2019;38(12):3193-3202. doi:10.1002/jum.15020

24. Jeong EY, Kim HL, Ha EJ, Park SY, Cho YJ, Han M. Computer-aided diagnosis system for thyroid nodules on ultrasonography: diagnostic performance and reproducibility based on the experience level of operators. Eur Radiol. 2019;29(4):1978-1985. doi:10.1007/s00330-018-5772-9

25. Li J, Sang T, Yu WH, et al. The value of S-Detect for the differential diagnosis of breast masses on ultrasound: a systematic review and pooled meta-analysis. Med Ultrason. 2020;22(2):211-219. doi:10.11152/mu-2402

Cancer Management and Research

Dovepress

\section{Publish your work in this journal}

Cancer Management and Research is an international, peer-reviewed open access journal focusing on cancer research and the optimal use of preventative and integrated treatment interventions to achieve improved outcomes, enhanced survival and quality of life for the cancer patient. The manuscript management system is completely online and includes a very quick and fair peer-review system, which is all easy to use. Visit http://www.dovepress.com/testimonials.php to read real quotes from published authors.

Submit your manuscript here: https://www.dovepress.com/cancer-management-and-research-journal 